\title{
Impact of 2017 ACC/AHA Guideline on Prevalence, Awareness, Treatment, Control and Risk Factors of Hypertension: a Population-based, Cross-sectional Study in Iran
}

\section{Fatemeh Sadeghi}

Karolinska Institutet https://orcid.org/0000-0002-0018-2954

\section{Bahman Cheraghian}

Alimentary Track Research Center

\section{Zahra Mohammadi}

Digestive Diseases Research Institute

\section{Sadaf G Sepanlou}

Digestive Diseases Research Institute

\section{Sahar Masoudi}

Digestive Diseases Research Institute

\section{Zahra Rahimi}

Ahvaz Jondishapour University of Medical Sciences

\section{Leila Danehchin}

Behbahan University of Medical Sciences

\section{Yousef Paridar}

Dzful University of Medical Sciences

\section{Farhad Abolnejadian}

Ahvaz Jundishapour University of Medical Sciences

\section{Mohammad Noori}

Abadan University of Medical Sciences

\section{Seyed Ali Mard}

Ahvaz Jundishapur University of Medical Sciences: Ahvaz Jondishapour University of Medical Sciences

\section{Ali Akbar Shayesteh}

Ahvaz Jundishapour University of Medical Sciences

Hossein Poustchi ( $\sim$ h.poustchi@gmail.com )

Liver and Pancreatobiliary Diseases Research Center, Digestive Diseases Research Institute, Tehran University of Medical Sciences, Tehran, Iran https://orcid.org/0000-0001-9058-9936 
Keywords: hypertension, Iran, 2017 ACC/AHA hypertension guideline, JNC8 hypertension guideline, risk factors, prevalence, awareness, treatment, control

Posted Date: September 9th, 2020

DOI: https://doi.org/10.21203/rs.3.rs-73452/v1

License: (c) (1) This work is licensed under a Creative Commons Attribution 4.0 International License. Read Full License 


\section{Abstract}

Background: In 2017, the American College of Cardiology/American Heart Association (ACC/AHA) provided a new guideline for hypertension prevention and management. We aimed to update the prevalence, awareness, control and risk factors of hypertension based on this guideline and to estimate the number of people who are eligible for non-pharmacologic and pharmacologic intervention.

Methods: This population-based, cross-sectional study was conducted in Khuzestan, a large province in the southwest of Iran. Comprehensive information about the potential risk factors of hypertension was collected and blood pressure, blood biomarkers, and anthropometric were measured. Moreover, the dietary pattern was evaluated in $10 \%$ of the participants, using a qualitative food frequency questionnaire.

Results: A total number of 30,506 individuals aged 20-65 years was included in this study. In comparison to previous guideline (JNC8), the prevalence of hypertension in Khuzestan dramatically increased by $27.04 \%$ after implementation of ACC/AHA (15.81\% vs $42.85 \%$ ), which was more dominant in the male population and the 50-59 age group. The sex and age adjustment of the hypertension prevalence was estimated to be $39.40 \%$. The number of individuals required antihypertensive treatment was slightly increased from $15.53 \%$ to $21.18 \%$; however, the level of awareness and control dramatically dropped by $23.17 \%$ \& $31.78 \%$, respectively. All hypertension-related risk factors remained significant after applying the new guideline; however, the strength of the association was reduced in the risk factors like age, waisthip ratio, body mass index, alcohol consumption, water pipe usage, and physical activity. Similarity, the association between hypertension and history of diabetes, and cardiovascular diseases were decreased by $38 \%$, and $62 \%$, respectively.

Conclusions: In the ACC/AHA guideline, a higher number of individuals with the pre-hypertension condition had been shifted into the hypertension category and the level of awareness, treatment, and control was dramatically fallen, which highlight a great need to expand the public health infrastructure for further managing the substantial increase in the public health burden of hypertension.

\section{Background}

Hypertension (HTN) is a major risk factor for non-communicable diseases such as cardiovascular diseases (CVD), stroke, and renal dysfunction. With 1.5 billion hypertensive people and 7.6 million HTNrelated death, this disease remains major public health globally $[1,2]$. In developing countries, the prevalence of HTN is increasing rapidly and it is estimated about three-fourths of the world's hypertensive population will be from these countries by 2025 [2]. Considering the high impact of HTN on public health, mainly on low resource settings with weak health systems, keeping updated about the prevalence of HTN as well as the attributed risk factors is essential to improve the approaches in controlling this disorder.

According to the eighth report of the joint national committee (JNC8) guideline, patients with systolic blood pressure (SBP) $\geq 140 \mathrm{mmHg}$ and/or diastolic blood pressure (DBP) $\geq 90 \mathrm{mmHg}$ have been 
diagnosed with HTN. Using this classification, the overall prevalence of HTN in Iran has been estimated to be $29.9 \%$ [3]. However, recently the 2017 American College of Cardiology (ACC)/American Heart Association (AHA) recommended $\geq 130 / 80 \mathrm{mmHg}$ as a new cut-off for HTN, indicating the estimations for prevalence, and the determinants of HTN are required to be updated in each region [4]. To date, few studies have evaluated the impact of suggested guideline and have reported shifting from JNC8 to 2017 ACC/AHA caused a significant increase in the prevalence of HTN and a drastic decline in awareness, treatment, and control of this disorder, which may impose a considerable impact on the health systems [5-7].

HTN is a multifactorial disease and several genetic, environmental, and behavioral factors contribute to manifest the disease [8]. Low physical activity and overweight, as well as unhealthy diet such as high salt intake, are the leading risk factors of HTN [9]; nevertheless, considerable variations between countries and even within the countries exist. Although this disease is usually asymptomatic and people are not aware of their condition, it is one of the most preventable disorder and modifications of the lifestyle could considerably prevent the progression of CVD and renal diseases in the future. In the ACC/AHA guideline, a higher number of individuals with the pre-hypertension condition had been shifted into the HTN category [4]. However, the impact of the 2017 ACC/AHA guideline on HTN-associated risk factors was not fully addressed and updating the major risk factors and their effect on HTN incidence is a prerequisite for effective prevention and awareness strategies in the future.

In Iran, CVD is the first cause of death [10]. Updating the local burden of the HTN as the major risk factor of CVD is important to set the health priority in the country. Moreover, primary prevention would have a major beneficial impact in terms of public health after defining the major risk factors of this disease. Therefore, we aimed to evaluate the prevalence of HTN according to updated guideline and evaluate the impact this guideline on awareness, treatment, and control as well as the major-associated risk factors in a large sample of participants in Khuzestan Comprehensive Health Study.

\section{Methods}

\section{Study design and study population}

This cross-sectional health survey was conducted based on the data of Khuzestan Comprehensive Health Study (KCHS). KCHS is a large population-based cross-section study with the purpose of evaluating the health status of the Iranian adults in Khuzestan. This study was performed in the period of October 2016 to November 2018 and the samples were selected using a multistage random sampling method. In the first stage of cluster sampling, the health centers and health houses within each county has been selected randomly (total 29 counties). According to the population of each county a total number of 1079 of clusters, consisted of 780 health centers in urban and 299 health houses in rural areas were chosen. Afterward, 30 individuals between 20-66 years old were selected within each cluster, using systematic random sampling. 
The individuals who had been selected in the sampling process and were residents of Khuzestan province for at least one year were invited to the study by a trained staff. We excluded those with mental, psychological or physical disabilities, or if they were reluctant to participate at any stage of the study. A written informed consent was obtained from all contributors before including them in the study.

\section{Blood Pressure and Anthropometric Measurements}

The SBP and DBP were measured twice with 10-minutes interval in a seated position using analogue Riester sphygmomanometers. In addition, the participants' height and weight were measured by Seca 206 body meter measuring tape and Seca 762 mechanical flat scale, respectively.

\section{Definitions}

According to the 2017 ACC/AHA guideline, HTN was defined if the SBP level was $\geq 130 \mathrm{mmHg}$ and/or the DBP level was $\geq 80 \mathrm{mmHg}$ and/or the participant takes antihypertensive medication [4]. The SBP between 120-129 mmHg and DBP $<80 \mathrm{mmHg}$ was considered as elevated blood pressure [4]. These thresholds were 140/90 for HTN and 120-139/80-89 for pre-hypertension based on the NCJ8 guideline [3]. Diabetes Mellitus (DM) was defined as fasting plasma glucose levels (FBS) $\geq 125 \mathrm{mg} / \mathrm{dL}$, previously diagnosed DM, or current intake of antidiabetic agents. We documented heart disease if the participant reported any history of myocardial infarction, angiography, or any other related heart diseases. The positive family history of HTN was considered if the participant reported a history of HTN in the firstdegree relatives.

Body mass index (BMI) was calculated by dividing weight (in kilograms) to height (in meters, squared) and further classified based on the WHO international BMI classification. Waist-to-hip ratio (WHR) was the ratio of waist circumference to hip circumference. The socioeconomic status was calculated by multiple correspondence analysis (MCA) with assets and was further presented in quartiles. Metabolic equivalent of task (MET) category was conducted based on the international physical activity questionnaire (IPAQ) guideline and was presented in tertial. Education was categorized according to the educational system in Iran.

Awareness was estimated based on the positive response of individual to self-reported HTN and treatment was considered if the participant takes antihypertensive medication. Moreover, the HTN control was defined in subjects with blood pressure $<130 / 80 \mathrm{mmHg}$ based on the $2017 \mathrm{ACC} / \mathrm{AHA}$ or < 90/140 $\mathrm{mmHg}$ based on the JNC8 after taking anti-HTN drugs.

\section{Laboratory measurements}

After overnight fasting, 15 milliliters peripheral blood was collected from each participant and was transferred to a research lab in a $4^{\circ} \mathrm{C}$ cold boxes within 3 hours. After separating the serum, the levels of FBS, total cholesterol, and high-density lipoproteins cholesterol (HDL) were measured.

\section{Dietary Intake Assessment}


The dietary habits were assessed for 2,830 of the participants, using non-quantitative food frequency questionnaire (FFQ) and the consumption of sodium, total fat, fiber, and carbohydrate were measured per week.

\section{Statistical analysis}

Using the Kolmogorov-Smirnov test, the normal distribution of the variables was evaluated. All continuous variables were presented as mean ( \pm standard deviation (SD)) and were compared between three independent groups (control, pre-hypertension or elevate blood pressure, HTN) using One-way ANOVA. The categorical variables were presented as absolute frequencies (percentages) and were compared with a chi-square test or Fisher's exact test, if required.

Multiple logistic regression was performed to identify the independent risk factors of pre-hypertension (or elevated blood pressure) and HTN in the studied population. The final model was adjusted with stablished risk factors of HTN such as sex, age, BMI, and physical activity. The graphs were presented by GraphPad Prism 7 (GraphPad Prism Software, San Diego, CA, USA) and the statistical analysis was performed using STATA/SE version 12.0 software (STATA Corp., TX, USA). p-value $<0.05$ was considered as statistically significant.

\section{Results}

\section{HTN-associated risk factors based on 2017 ACC/AHA guideline}

A total number of 30,506 individuals aged 20-65 years was included in this study. Using $2017 \mathrm{ACC} / \mathrm{AHA}$ cut-off the overall prevalence of elevated blood pressure and HTN were 1,950 (6.41\%) \& 13,036 (42.85\%), respectively, which changed to $7.09 \%$ and $39.40 \%$ after sex and age adjustment. The mean ( $\pm S D)$ age was 41.71 ( \pm 11.89$)$, which was significantly higher in hypertensive people compared to the controls $(P=$ $0.000)$. In the studied population, women were prominent $(64.26 \%)$; however, they were less likely to manifest HTN compared to men (50.03\% vs $38.70 \%)$. Other independent protective risk factors were being Bakhtiary, getting married, having higher education level, doing more physical activity, and smoking, whereas being Arab, having high WHR and BMI, drinking alcohol, and having a family history of HTN were positively associated with an increased risk of HTN (Table 1). The risk factors associated with elevated blood pressure were age, gender, marital status, BMI, alcohol intake, physical activity, and family history of HTN. 
Table 1

Adjusted odds ratios and $95 \%$ confidence intervals for association between studied variables and hypertension among Iranian: NCJ8 guideline vs 2017 ACC/AHA guideline

\begin{tabular}{|c|c|c|c|c|c|c|c|c|}
\hline \multirow[t]{3}{*}{ Variables } & \multicolumn{4}{|c|}{ NCJ8 guideline } & \multicolumn{4}{|c|}{2017 ACC/AHA guideline } \\
\hline & \multicolumn{2}{|c|}{$\begin{array}{l}\text { Pre- } \\
\text { hypertension vs } \\
\text { control }\end{array}$} & \multicolumn{2}{|c|}{$\begin{array}{l}\text { Hypertension vs } \\
\text { control }\end{array}$} & \multicolumn{2}{|c|}{$\begin{array}{l}\text { Elevated BP vs } \\
\text { control }\end{array}$} & \multicolumn{2}{|c|}{$\begin{array}{l}\text { Hypertension } \\
\text { vs control }\end{array}$} \\
\hline & $\begin{array}{l}\text { OR } \\
(95 \% \\
\text { Cl) }\end{array}$ & $\begin{array}{l}\mathrm{p}- \\
\text { value }\end{array}$ & $\begin{array}{l}\text { OR } \\
(95 \% \\
\text { Cl) }\end{array}$ & $\begin{array}{l}\mathrm{p}- \\
\text { value }\end{array}$ & $\begin{array}{l}\text { OR } \\
(95 \% \\
\text { Cl) }\end{array}$ & $\begin{array}{l}\mathrm{p}- \\
\text { value }\end{array}$ & $\begin{array}{l}\text { OR } \\
(95 \% \\
\text { Cl) }\end{array}$ & $\begin{array}{l}\mathrm{p} \text { - } \\
\text { value }\end{array}$ \\
\hline Age group & 1 & 0.087 & 1 & 0.000 & 1 & 0.999 & 1 & 0.001 \\
\hline $20-29$ & 1.07 & 0.000 & 1.53 & 0.000 & 1.00 & & 1.14 & 0.000 \\
\hline $30-39$ & $1.16)$ & 0.000 & $1.80)$ & 0.000 & $1.15)$ & 0.000 & $1.23)^{-}$ & 0.000 \\
\hline $40-49$ & 1.51 & 0.000 & 4.32 & 0.000 & 1.26 & 0.000 & 1.98 & 0.000 \\
\hline $50-59$ & $1.64)$ & & $5.05)$ & & $1.46)$ & & $2.15)$ & \\
\hline \multirow[t]{2}{*}{$>60$} & $\begin{array}{l}2.11 \\
(1.94- \\
2.30)\end{array}$ & & $\begin{array}{l}11.49 \\
(9.84- \\
13.41)\end{array}$ & & $\begin{array}{l}1.67 \\
(1.43- \\
1.95)\end{array}$ & & $\begin{array}{l}3.66 \\
(3.37- \\
3.98)\end{array}$ & \\
\hline & $\begin{array}{l}2.60 \\
(2.31- \\
2.93)\end{array}$ & & $\begin{array}{l}20.22 \\
(17.00- \\
24.05)\end{array}$ & & $\begin{array}{l}1.85 \\
(1.49- \\
2.29)\end{array}$ & & $\begin{array}{l}5.49 \\
(4.91- \\
6.14)\end{array}$ & \\
\hline Gender & 1 & 0.000 & 1 & 0.000 & 1 & 0.000 & 1 & 0.000 \\
\hline Male & \multirow{2}{*}{\multicolumn{2}{|c|}{$\begin{array}{l}0.51 \\
(0.48- \\
0.53)\end{array}$}} & \multirow{2}{*}{\multicolumn{2}{|c|}{$\begin{array}{l}0.59 \\
(0.55- \\
0.64)\end{array}$}} & \multirow{2}{*}{\multicolumn{2}{|c|}{$\begin{array}{l}0.46 \\
(0.42- \\
0.51)\end{array}$}} & \multirow{2}{*}{\multicolumn{2}{|c|}{$\begin{array}{l}0.53 \\
(0.51- \\
0.56)\end{array}$}} \\
\hline Female & & & & & & & & \\
\hline Marital status & 1 & 0.000 & 1 & 0.448 & 1 & 0.001 & 1 & 0.004 \\
\hline Single & \multirow{2}{*}{$\begin{array}{l}0.83 \\
(0.76- \\
0.90)\end{array}$} & \multirow{2}{*}{$\begin{array}{l}0.501 \\
0.367\end{array}$} & \multirow{2}{*}{$\begin{array}{l}1.07 \\
(0.90- \\
1.26)\end{array}$} & & \multirow{2}{*}{$\begin{array}{l}0.77 \\
(0.66- \\
0.90)\end{array}$} & \multirow[t]{2}{*}{0.162} & \multirow{2}{*}{$\begin{array}{l}0.88 \\
(0.80- \\
0.96)\end{array}$} & \\
\hline Married & & & & 0.010 & & & & .446 \\
\hline Divorced & \multirow{2}{*}{\multicolumn{2}{|c|}{$\begin{array}{l}0.92 \\
(0.71- \\
1.18)\end{array}$}} & \multirow{2}{*}{\multicolumn{2}{|c|}{$\begin{array}{l}1.02 \\
(0.69- \\
1.51)\end{array}$}} & \multirow{2}{*}{\multicolumn{2}{|c|}{$\begin{array}{l}0.87 \\
(0.54- \\
1.38)\end{array}$}} & \multirow{2}{*}{\multicolumn{2}{|c|}{$\begin{array}{l}0.91 \\
(0.71- \\
1.17)\end{array}$}} \\
\hline Widowed & & & & & & & & \\
\hline & \multicolumn{2}{|l|}{$\begin{array}{l}0.92 \\
(0.78- \\
1.10)\end{array}$} & \multicolumn{2}{|l|}{$\begin{array}{l}1.35 \\
(1.07- \\
1.70)\end{array}$} & \multicolumn{2}{|l|}{$\begin{array}{l}0.79 \\
(0.57- \\
1.10)\end{array}$} & \multicolumn{2}{|l|}{$\begin{array}{l}1.06 \\
(0.91- \\
1.25)\end{array}$} \\
\hline
\end{tabular}




\begin{tabular}{|c|c|c|c|c|c|c|c|c|}
\hline \multirow[t]{3}{*}{ Variables } & \multicolumn{4}{|c|}{ NCJ8 guideline } & \multicolumn{4}{|c|}{2017 ACC/AHA guideline } \\
\hline & \multicolumn{2}{|c|}{$\begin{array}{l}\text { Pre- } \\
\text { hypertension vs } \\
\text { control }\end{array}$} & \multicolumn{2}{|c|}{$\begin{array}{l}\text { Hypertension vs } \\
\text { control }\end{array}$} & \multicolumn{2}{|c|}{$\begin{array}{l}\text { Elevated BP vs } \\
\text { control }\end{array}$} & \multicolumn{2}{|c|}{$\begin{array}{l}\text { Hypertension } \\
\text { vs control }\end{array}$} \\
\hline & $\begin{array}{l}\text { OR } \\
(95 \% \\
\mathrm{Cl})\end{array}$ & $\begin{array}{l}\mathrm{p}- \\
\text { value }\end{array}$ & $\begin{array}{l}\text { OR } \\
(95 \% \\
\mathrm{Cl})\end{array}$ & $\begin{array}{l}\mathrm{p}- \\
\text { value }\end{array}$ & $\begin{array}{l}\text { OR } \\
(95 \% \\
\mathrm{Cl})\end{array}$ & $\begin{array}{l}\mathrm{p}- \\
\text { value }\end{array}$ & $\begin{array}{l}\text { OR } \\
(95 \% \\
\text { Cl) }\end{array}$ & $\begin{array}{l}\mathrm{p}- \\
\text { value }\end{array}$ \\
\hline \multirow{3}{*}{$\begin{array}{l}\text { Socioeconomic } \\
\text { status } \\
\text { Q1 }\end{array}$} & 1 & 0.739 & 1 & 0.755 & 1 & 0.687 & 1 & 0.980 \\
\hline & \multirow{2}{*}{$\begin{array}{l}1.01 \\
(0.94- \\
1.09)\end{array}$} & 0.010 & \multirow{2}{*}{$\begin{array}{l}0.98 \\
(0.89- \\
1.09)\end{array}$} & 0.000 & \multirow{2}{*}{$\begin{array}{l}1.03 \\
(0.90- \\
1.18)\end{array}$} & 0.360 & \multirow{2}{*}{$\begin{array}{l}1.0 \\
(0.93- \\
1.07)\end{array}$} & 0.000 \\
\hline & & 0.001 & & 0.000 & & 0.709 & & 0.000 \\
\hline Q3 & $\begin{array}{l}0.91 \\
(0.84- \\
0.98)\end{array}$ & & $\begin{array}{l}0.81 \\
(0.73- \\
0.90)\end{array}$ & & $\begin{array}{l}0.94 \\
(0.82- \\
1.08)\end{array}$ & & $\begin{array}{l}0.88 \\
(0.82- \\
0.94)\end{array}$ & \\
\hline Q4 & \multicolumn{2}{|l|}{$\begin{array}{l}0.88 \\
(0.82- \\
0.95)\end{array}$} & \multicolumn{2}{|l|}{$\begin{array}{l}0.78 \\
(0.70- \\
0.86)\end{array}$} & \multicolumn{2}{|l|}{$\begin{array}{l}0.97 \\
(0.85- \\
1.12)\end{array}$} & \multicolumn{2}{|l|}{$\begin{array}{l}0.83 \\
(0.77- \\
0.89)\end{array}$} \\
\hline Education & 1 & 0.077 & 1 & 0.000 & 1 & 0.616 & 1 & 0.000 \\
\hline Illiterate & \multirow{2}{*}{$\begin{array}{l}0.93 \\
(0.85- \\
1.01)\end{array}$} & & 0.76 & 0.000 & \multirow{2}{*}{$\begin{array}{l}0.97 \\
(0.82- \\
1.12)\end{array}$} & & \multirow{2}{*}{$\begin{array}{l}0.84 \\
(0.78- \\
0.91)\end{array}$} & 0.000 \\
\hline Elementary & & $\begin{array}{l}0.000 \\
0.000\end{array}$ & $\begin{array}{l}(0.68- \\
0.84)\end{array}$ & 0.000 & & $\begin{array}{l}0.4 / 5 \\
0.380\end{array}$ & & 0.000 \\
\hline $\begin{array}{l}\text { Guidance } \\
\text { school }\end{array}$ & $\begin{array}{l}0.82 \\
(0.75- \\
0.91)\end{array}$ & & $\begin{array}{l}0.67 \\
(0.59- \\
0.76)\end{array}$ & 0.000 & $\begin{array}{l}0.98 \\
(0.83- \\
1.17)\end{array}$ & & $\begin{array}{l}0.73 \\
(0.66- \\
0.80)\end{array}$ & 0.000 \\
\hline \multirow{3}{*}{$\begin{array}{l}\text { High school } \\
\text { Universities }\end{array}$} & \multirow{2}{*}{\multicolumn{2}{|c|}{$\begin{array}{l}0.84 \\
(0.77- \\
0.91)\end{array}$}} & \multirow{2}{*}{\multicolumn{2}{|c|}{$\begin{array}{l}0.61 \\
(0.54- \\
0.68)\end{array}$}} & \multirow{2}{*}{\multicolumn{2}{|c|}{$\begin{array}{l}0.94 \\
(0.80- \\
1.11)\end{array}$}} & \multirow{2}{*}{\multicolumn{2}{|c|}{$\begin{array}{l}0.72 \\
(0.67- \\
0.78)\end{array}$}} \\
\hline & & & & & & & & \\
\hline & \multicolumn{2}{|l|}{$\begin{array}{l}0.78 \\
(0.70- \\
0.86)\end{array}$} & \multicolumn{2}{|l|}{$\begin{array}{l}0.54 \\
(0.46- \\
0.63)\end{array}$} & \multicolumn{2}{|l|}{$\begin{array}{l}0.92 \\
(0.76- \\
1.11)\end{array}$} & \multicolumn{2}{|l|}{$\begin{array}{l}0.66 \\
(0.59- \\
0.73)\end{array}$} \\
\hline
\end{tabular}




\begin{tabular}{|c|c|c|c|c|c|c|c|c|}
\hline \multirow[t]{3}{*}{ Variables } & \multicolumn{4}{|c|}{ NCJ8 guideline } & \multicolumn{4}{|c|}{2017 ACC/AHA guideline } \\
\hline & \multicolumn{2}{|c|}{$\begin{array}{l}\text { Pre- } \\
\text { hypertension vs } \\
\text { control }\end{array}$} & \multicolumn{2}{|c|}{$\begin{array}{l}\text { Hypertension vs } \\
\text { control }\end{array}$} & \multicolumn{2}{|c|}{$\begin{array}{l}\text { Elevated BP vs } \\
\text { control }\end{array}$} & \multicolumn{2}{|c|}{$\begin{array}{l}\text { Hypertension } \\
\text { vs control }\end{array}$} \\
\hline & $\begin{array}{l}\text { OR } \\
(95 \% \\
\text { Cl) }\end{array}$ & $\begin{array}{l}\mathrm{p}- \\
\text { value }\end{array}$ & $\begin{array}{l}\text { OR } \\
(95 \% \\
\text { Cl) }\end{array}$ & $\begin{array}{l}\mathrm{p}- \\
\text { value }\end{array}$ & $\begin{array}{l}\text { OR } \\
(95 \% \\
\mathrm{Cl})\end{array}$ & $\begin{array}{l}\mathrm{p}- \\
\text { value }\end{array}$ & $\begin{array}{l}\text { OR } \\
(95 \% \\
\mathrm{Cl})\end{array}$ & $\begin{array}{l}\mathrm{p}- \\
\text { value }\end{array}$ \\
\hline Ethnicity & 1 & 0.000 & 1 & 0.000 & 1 & 0.306 & 1 & 0.000 \\
\hline Fars & 1.17 & 0.000 & 1.22 & 0.003 & 1.07 & 0.775 & 1.21 & 0.000 \\
\hline Arab & $1.26)$ & 0.060 & $1.34)$ & $\begin{array}{l}0.151 \\
0102\end{array}$ & $1.23)$ & 0.110 & $1.29)$ & 0.763 \\
\hline $\begin{array}{l}\text { Bakhtiary } \\
\text { Lor }\end{array}$ & $\begin{array}{l}0.81 \\
(0.75- \\
0.88)\end{array}$ & & $\begin{array}{l}0.84 \\
(0.75- \\
0.94)\end{array}$ & & $\begin{array}{l}1.02 \\
(0.88- \\
1.19)\end{array}$ & & $\begin{array}{l}0.79 \\
(0.73- \\
0.85)\end{array}$ & \\
\hline \multirow[t]{2}{*}{ Others } & $\begin{array}{l}0.90 \\
(0.81- \\
1.00)\end{array}$ & & $\begin{array}{l}1.11 \\
(0.96- \\
1.28)\end{array}$ & & $\begin{array}{l}0.85 \\
(0.70- \\
1.04)\end{array}$ & & $\begin{array}{l}0.98 \\
(0.89- \\
1.09)\end{array}$ & \\
\hline & $\begin{array}{l}0.86 \\
(0.64- \\
1.17)\end{array}$ & & $\begin{array}{l}0.45 \\
(0.27- \\
0.76)\end{array}$ & & $\begin{array}{l}0.82 \\
(0.46- \\
1.45)\end{array}$ & & $\begin{array}{l}0.75 \\
(0.55- \\
1.01)\end{array}$ & \\
\hline Waist-hip ratio & 1 & 0.001 & 1 & 0.000 & 1 & 0.738 & 1 & 0.000 \\
\hline WHR $<0.85$ & \multirow{2}{*}{$\begin{array}{l}1.15 \\
(1.06- \\
1.24)\end{array}$} & 0.000 & \multirow{2}{*}{$\begin{array}{l}1.43 \\
(1.26- \\
1.63)\end{array}$} & 0.000 & \multirow{2}{*}{$\begin{array}{l}1.02 \\
(0.89- \\
1.18)\end{array}$} & 0.820 & \multirow{2}{*}{$\begin{array}{l}1.23 \\
(1.14- \\
1.33)\end{array}$} & 0.000 \\
\hline $0.85 \leq W H R<$ & & 0.000 & & 0.000 & & 0.838 & & 0.000 \\
\hline $\begin{array}{l}0.90 \leq W H R< \\
0.95\end{array}$ & $\begin{array}{l}1.24 \\
(1.14- \\
1.34)\end{array}$ & & $\begin{array}{l}1.85 \\
(1.63- \\
2.10)\end{array}$ & & $\begin{array}{l}0.98 \\
(0.85- \\
1.14)\end{array}$ & & $\begin{array}{l}1.42 \\
(1.31- \\
1.53)\end{array}$ & \\
\hline WHR $\geq 0.95$ & $\begin{array}{l}1.25 \\
(1.15- \\
1.35)\end{array}$ & & $\begin{array}{l}2.38 \\
(2.11- \\
2.69)\end{array}$ & & $\begin{array}{l}1.02 \\
(0.87- \\
1.18)\end{array}$ & & $\begin{array}{l}1.57 \\
(1.45- \\
1.70)\end{array}$ & \\
\hline \multirow{3}{*}{$\begin{array}{l}\text { BMI } \\
\text { Normal (18.6- } \\
25)\end{array}$} & 1 & 0.000 & 1 & $\begin{array}{l}0.000 \\
0.000\end{array}$ & 1 & $\begin{array}{l}0.008 \\
0.000\end{array}$ & 1 & 0.000 \\
\hline & \multirow{2}{*}{$\begin{array}{l}0.65 \\
(0.54- \\
0.78)\end{array}$} & 0.000 & \multirow{2}{*}{$\begin{array}{l}0.49 \\
(0.374- \\
0.70)\end{array}$} & & \multirow{2}{*}{$\begin{array}{l}0.64 \\
(0.46- \\
0.89)\end{array}$} & \multirow{2}{*}{0.000} & \multirow{2}{*}{$\begin{array}{l}0.62 \\
(0.52- \\
0.74)\end{array}$} & 0.000 \\
\hline & & 0.000 & & 0.000 & & & & 0.000 \\
\hline $\begin{array}{l}\text { Underweight } \\
(\leq 18.5)\end{array}$ & \multirow{2}{*}{\multicolumn{2}{|c|}{$\begin{array}{l}1.38 \\
(1.29- \\
1.48)\end{array}$}} & \multirow{2}{*}{\multicolumn{2}{|c|}{$\begin{array}{l}1.74 \\
(1.58- \\
1.92)\end{array}$}} & \multirow{2}{*}{\multicolumn{2}{|c|}{$\begin{array}{l}1.30 \\
(1.15- \\
1.46)\end{array}$}} & \multirow{2}{*}{\multicolumn{2}{|c|}{$\begin{array}{l}1.49 \\
(1.40- \\
1.59)\end{array}$}} \\
\hline Overweight & & & & & & & & \\
\hline Obese $(>30)$ & \multicolumn{2}{|l|}{$\begin{array}{l}1.84 \\
(1.72- \\
1.97)\end{array}$} & \multicolumn{2}{|l|}{$\begin{array}{l}2.93 \\
(2.64- \\
3.25)\end{array}$} & \multicolumn{2}{|l|}{$\begin{array}{l}1.44 \\
(1.26- \\
1.64)\end{array}$} & \multicolumn{2}{|l|}{$\begin{array}{l}2.23 \\
(2.08- \\
2.39)\end{array}$} \\
\hline
\end{tabular}




\begin{tabular}{|c|c|c|c|c|c|c|c|c|}
\hline \multirow[t]{3}{*}{ Variables } & \multicolumn{4}{|c|}{ NCJ8 guideline } & \multicolumn{4}{|c|}{2017 ACC/AHA guideline } \\
\hline & \multicolumn{2}{|c|}{$\begin{array}{l}\text { Pre- } \\
\text { hypertension vs } \\
\text { control }\end{array}$} & \multicolumn{2}{|c|}{$\begin{array}{l}\text { Hypertension vs } \\
\text { control }\end{array}$} & \multicolumn{2}{|c|}{$\begin{array}{l}\text { Elevated BP vs } \\
\text { control }\end{array}$} & \multicolumn{2}{|c|}{$\begin{array}{l}\text { Hypertension } \\
\text { vs control }\end{array}$} \\
\hline & $\begin{array}{l}\text { OR } \\
(95 \% \\
\text { Cl) }\end{array}$ & $\begin{array}{l}\mathrm{p}- \\
\text { value }\end{array}$ & $\begin{array}{l}\text { OR } \\
(95 \% \\
\text { Cl) }\end{array}$ & $\begin{array}{l}\mathrm{p}- \\
\text { value }\end{array}$ & $\begin{array}{l}\text { OR } \\
(95 \% \\
\text { Cl) }\end{array}$ & $\begin{array}{l}\mathrm{p}- \\
\text { value }\end{array}$ & $\begin{array}{l}\text { OR } \\
(95 \% \\
\text { Cl) }\end{array}$ & $\begin{array}{l}\mathrm{p} \text { - } \\
\text { value }\end{array}$ \\
\hline \multirow{3}{*}{$\begin{array}{l}\text { Smoking } \\
\text { No } \\
\text { Current }\end{array}$} & 1 & \multirow{4}{*}{$\begin{array}{l}0.001 \\
0.367\end{array}$} & 1 & \multirow{4}{*}{$\begin{array}{l}0.000 \\
0.109\end{array}$} & 1 & \multirow{4}{*}{$\begin{array}{l}0.571 \\
0.910\end{array}$} & 1 & 0.000 \\
\hline & \multirow{2}{*}{$\begin{array}{l}0.84 \\
(0.76- \\
0.93)\end{array}$} & & \multirow{2}{*}{$\begin{array}{l}0.76 \\
(0.66- \\
0.89)\end{array}$} & & \multirow{2}{*}{$\begin{array}{l}0.95 \\
(0.80- \\
1.13)\end{array}$} & & 0.79 & \multirow[t]{3}{*}{0.879} \\
\hline & & & & & & & $\begin{array}{l}(0.72- \\
0.88)\end{array}$ & \\
\hline Former & $\begin{array}{l}0.93 \\
(0.79- \\
1.09)\end{array}$ & & $\begin{array}{l}1.17 \\
(0.97- \\
1.42)\end{array}$ & & $\begin{array}{l}0.98 \\
(0.75- \\
1.29)\end{array}$ & & $\begin{array}{l}0.99 \\
(0.85- \\
1.15)\end{array}$ & \\
\hline \multirow{4}{*}{$\begin{array}{l}\text { Alcohol } \\
\text { No } \\
\text { Current } \\
\text { Former }\end{array}$} & 1 & \multirow{4}{*}{$\begin{array}{l}0.069 \\
0.095\end{array}$} & 1 & \multirow{4}{*}{$\begin{array}{l}0.227 \\
0.041\end{array}$} & \multirow{4}{*}{$\begin{array}{l}1.59 \\
(1.08- \\
2.35) \\
1.07 \\
(0.71- \\
1.62)\end{array}$} & \multirow{4}{*}{$\begin{array}{l}0.019 \\
0.744\end{array}$} & 1 & \multirow{4}{*}{$\begin{array}{l}0.191 \\
0.027\end{array}$} \\
\hline & \multirow{2}{*}{$\begin{array}{l}1.27 \\
(0.98- \\
1.63)\end{array}$} & & \multirow{2}{*}{$\begin{array}{l}1.31 \\
(0.84- \\
2.03)\end{array}$} & & & & \multirow{2}{*}{$\begin{array}{l}1.19 \\
(0.92- \\
1.54)\end{array}$} & \\
\hline & & & & & & & & \\
\hline & $\begin{array}{l}1.22 \\
(0.97- \\
1.55)\end{array}$ & & $\begin{array}{l}1.44 \\
(1.01- \\
2.07)\end{array}$ & & & & $\begin{array}{l}1.30 \\
(1.03- \\
1.64)\end{array}$ & \\
\hline \multirow{2}{*}{$\begin{array}{l}\text { Water pipe } \\
\text { (hookah) }\end{array}$} & 1 & \multirow{5}{*}{$\begin{array}{l}0.096 \\
0.070\end{array}$} & 1 & \multirow{5}{*}{$\begin{array}{l}0.028 \\
0.042\end{array}$} & \multirow{5}{*}{$\begin{array}{l}1.08 \\
(0.85- \\
1.37) \\
1.25 \\
(0.88- \\
1.79)\end{array}$} & \multirow{5}{*}{$\begin{array}{l}0.538 \\
0.208\end{array}$} & 1 & \multirow{5}{*}{$\begin{array}{l}0.025 \\
0.047\end{array}$} \\
\hline & \multirow{2}{*}{$\begin{array}{l}1.12 \\
(0.98- \\
1.28)\end{array}$} & & \multirow{3}{*}{$\begin{array}{l}1.27 \\
(1.03- \\
1.58)\end{array}$} & & & & \multirow{2}{*}{$\begin{array}{l}1.16 \\
(1.02- \\
1.33)\end{array}$} & \\
\hline TNO & & & & & & & & \\
\hline Current & \multirow{2}{*}{$\begin{array}{l}1.21 \\
(0.98- \\
1.50)\end{array}$} & & & & & & 122 & \\
\hline Former & & & $\begin{array}{l}(1.01- \\
1.87)\end{array}$ & & & & $\begin{array}{l}(1.00- \\
1.51)\end{array}$ & \\
\hline Physical & 1 & 0.000 & 1 & 0.000 & 0.85 & 0.004 & 1 & 0.000 \\
\hline & 0.87 & 0.000 & 0.76 & 0.000 & $0.95)$ & 0.000 & 0.83 & 0.000 \\
\hline & $0.93)$ & & $0.82)$ & & 0.79 & & $0.88)$ & \\
\hline T3 & $\begin{array}{l}0.88 \\
(0.82- \\
0.94)\end{array}$ & & $\begin{array}{l}0.75 \\
(0.68- \\
0.83)\end{array}$ & & $0.90)$ & & $\begin{array}{l}0.85 \\
(0.79- \\
0.91)\end{array}$ & \\
\hline Family history & 1 & 0.000 & 1 & 0.000 & 1.13 & 0.014 & 1 & 0.000 \\
\hline $\begin{array}{l}\text { No } \\
\text { Yes }\end{array}$ & $\begin{array}{l}1.18 \\
(1.12- \\
1.25)\end{array}$ & & $\begin{array}{l}1.81 \\
(1.68- \\
1.96)\end{array}$ & & $1.25)$ & & $\begin{array}{l}1.36 \\
(1.29- \\
1.43)\end{array}$ & \\
\hline
\end{tabular}




\begin{tabular}{|c|c|c|c|c|c|c|c|c|}
\hline \multirow[t]{3}{*}{ Variables } & \multicolumn{4}{|c|}{ NCJ8 guideline } & \multicolumn{4}{|c|}{2017 ACC/AHA guideline } \\
\hline & \multicolumn{2}{|c|}{$\begin{array}{l}\text { Pre- } \\
\text { hypertension vs } \\
\text { control }\end{array}$} & \multicolumn{2}{|c|}{$\begin{array}{l}\text { Hypertension vs } \\
\text { control }\end{array}$} & \multicolumn{2}{|c|}{$\begin{array}{l}\text { Elevated BP vs } \\
\text { control }\end{array}$} & \multicolumn{2}{|c|}{$\begin{array}{l}\text { Hypertension } \\
\text { vs control }\end{array}$} \\
\hline & $\begin{array}{l}\text { OR } \\
(95 \% \\
\text { Cl) }\end{array}$ & $\begin{array}{l}\text { p- } \\
\text { value }\end{array}$ & $\begin{array}{l}\text { OR } \\
(95 \% \\
\text { Cl) }\end{array}$ & $\begin{array}{l}\text { p- } \\
\text { value }\end{array}$ & $\begin{array}{l}\text { OR } \\
(95 \% \\
\mathrm{Cl})\end{array}$ & $\begin{array}{l}\text { p- } \\
\text { value }\end{array}$ & $\begin{array}{l}\text { OR } \\
(95 \% \\
\text { Cl) }\end{array}$ & $\begin{array}{l}\mathrm{p} \text { - } \\
\text { value }\end{array}$ \\
\hline DM & 1 & 0.000 & 1 & 0.000 & 1.35 & 0.000 & 1 & 0.000 \\
\hline $\begin{array}{l}\text { No } \\
\text { Yes }\end{array}$ & \multicolumn{2}{|l|}{$\begin{array}{l}1.36 \\
(1.25- \\
1.47)\end{array}$} & \multicolumn{2}{|l|}{$\begin{array}{l}1.99 \\
(1.81- \\
2.18)\end{array}$} & $\begin{array}{l}(1.17- \\
1.56)\end{array}$ & & \multicolumn{2}{|l|}{$\begin{array}{l}1.62 \\
(1.50- \\
1.74)\end{array}$} \\
\hline \multirow{2}{*}{$\begin{array}{l}\text { Cardiovascular } \\
\text { disease } \\
\text { No } \\
\text { Yes }\end{array}$} & 1 & 0.260 & 1 & 0.000 & 1 & 0.510 & 1 & 0.000 \\
\hline & \multicolumn{2}{|l|}{$\begin{array}{l}1.08 \\
(0.94- \\
1.24)\end{array}$} & \multicolumn{2}{|l|}{$\begin{array}{l}2.10 \\
(1.83- \\
2.41)\end{array}$} & \multicolumn{2}{|l|}{$\begin{array}{l}1.08 \\
(0.85- \\
1.38)\end{array}$} & \multicolumn{2}{|l|}{$\begin{array}{l}1.49 \\
(1.32- \\
1.68)\end{array}$} \\
\hline \multirow{2}{*}{$\begin{array}{l}\text { Cholesterol } \\
(\mathrm{mg} / \mathrm{dL}) \\
<240 \\
>240\end{array}$} & 1 & 0.000 & 1 & 0.000 & 1 & 0.002 & 1 & 0.000 \\
\hline & \multicolumn{2}{|l|}{$\begin{array}{l}1.16 \\
(1.07- \\
1.25)\end{array}$} & $\begin{array}{l}1.21 \\
(1.10- \\
1.34)\end{array}$ & & $\begin{array}{l}1.24 \\
(1.08- \\
1.42)\end{array}$ & & $\begin{array}{l}1.17 \\
(1.08- \\
1.25)\end{array}$ & \\
\hline $\mathrm{HDL}(\mathrm{mg} / \mathrm{dL})$ & 1 & 0.079 & 1 & 0.009 & 1 & 0.128 & 1 & 0.039 \\
\hline $\begin{array}{l}<30 \\
>30\end{array}$ & \multicolumn{2}{|l|}{$\begin{array}{l}0.82 \\
(0.66- \\
1.02)\end{array}$} & \multicolumn{2}{|l|}{$\begin{array}{l}0.68 \\
(0.51- \\
0.90)\end{array}$} & \multicolumn{2}{|l|}{$\begin{array}{l}0.76 \\
(0.53- \\
1.08)\end{array}$} & $\begin{array}{l}0.80 \\
(0.65- \\
0.99)\end{array}$ & \\
\hline \multicolumn{9}{|c|}{ Odds ratios were adjusted for age, gender, $\mathrm{BMI}$, and physical activity } \\
\hline \multicolumn{9}{|c|}{$\begin{array}{l}\text { JNC8: eighth report of the joint national committee, ACC/AHA: } 2017 \text { American College of } \\
\text { Cardiology/American Heart Association }\end{array}$} \\
\hline \multicolumn{9}{|c|}{$\begin{array}{l}\text { BP: blood pressure, BMI: body mass index, CVD: cardiovascular diseases, OR: odds ratio, Cl: } \\
\text { confidence interval, Q: quartile, T: tertile }\end{array}$} \\
\hline Bold values indic & tatistica & ignificar & & & & & & \\
\hline
\end{tabular}

Among biological risk factors, a high level of cholesterol was positivity associated with both elevated blood pressure and HTN, while a significant lower level of HDL was observed in the hypertensive people only (Table 1$)$.

In the nutrient assessment, we found no significant differences in the dietary habits of hypertensive and healthy group. In the sex stratification analysis, high fat intake was associated with a two-fold increased risk of HTN among males. In addition, a lower intake of salt and carbohydrate seems to be protective against HTN in the age group of 20-29 (data not shown). 


\section{Prevalence, and the determinants of HTN: 2017 ACC/AHA versus JNC8 hypertension guidelines}

According to JNC8, the overall prevalence of HTN was $15.81 \%$, which considerably increased by $27.04 \%$ after adopting the $2017 \mathrm{ACC} / \mathrm{AHA}$ guideline (42.85\%) (Fig. 1a). This difference was more dominant in male $(17.8 \%$ vs $50.3 \%)$ and individuals between $50-59$ years old $(29.54-59.74 \%)$ (Fig. $1 \mathrm{~b})$. Although $3.82 \%$ of the $20-29$ age group were diagnosed with HTN based on JNC8, this level increased to $26.02 \%$ by applying the new guideline.

Moreover, the association between HTN and 60-65 aged group was 20.9-fold higher than HTN and 2029 people; nevertheless, the association reduced to 5.6-fold after declining the blood pressure cut-off point by $10 \mathrm{mmHg}$ to $130 / 80 \mathrm{mmHg}$. According to the ACC/AHA classification, $22.17 \%$ of underweight precipitants had HTN, while only $4.75 \%$ of this group were diagnosed with HTN previously. Moreover, the magnitude of the odds ratio was reduced from $2.22-1.43 \%$ in obese participants in comparison to subjects with normal BMI level.

Among HTN-identified people, 45.94\% were aware, and among those eligible for pharmacologic intervention $19.40 \%$ received anti-hyper medication, which controlled the HTN in $60.72 \%$ of subjects. Nevertheless, after implementation of 2017 ACC/AHA, the rate of awareness, treatment, and control dropped to $22.77 \%, 14.22 \%$, and $28.94 \%$, respectively (Fig. 1 C).

\section{Discussion}

After implementation of the 2017 ACC/AHA guideline, the prevalence of HTN in the Khuzestan province dramatically increased by $27.04 \%$ and the level of awareness and control dropped by $23.17 \% \& 31.78 \%$, respectively. Accordingly, the overall prevalence of HTN was $42.85 \%$ and about $77.23 \%$ of hypertensive adult were not aware of their condition. Among those who were eligible for HTN therapy $14.22 \%$ received drug, which controlled the HTN in $28.94 \%$ of them. All hypertension-related risk factors remained significant after applying the new guideline; however, the strength of the association was reduced in the risk factors like WHR, BMI, alcohol consumption, water pipe usage and physical activity. Moreover, the association between HTN and history of DM, and CAD was reduced by $38 \%$, and $62 \%$ respectively.

In order to reduce the major adverse of CVD events, ACC/AHA has recommended a lower threshold of SBP and DBS (130/80 $\mathrm{mm} \mathrm{Hg}$ ) for diagnosis of HTN compared to what had been suggested earlier (140/90 $\mathrm{mm} \mathrm{Hg})$; however, there is a debate about the proposed HTN cut-off and CVD benefit. Although some studies reported reducing the SBP to $120 \mathrm{~mm} \mathrm{Hg}$ level significantly decreased the risk of CVD [11], a more substantial reduction rate was observed for 130-139 mm Hg versus $<120 \mathrm{~mm} \mathrm{Hg}[12,13]$. Conversely, a recent systematic review and meta-analysis study found no CVD benefit from additional blood pressure lowering if the SBP at baseline was $<140 \mathrm{~mm} \mathrm{Hg}$ [14] and the authors further declared implementation of this guideline caused a significant increase in the proportion of adult diagnosed with HTN, with no noticeable effect on the reducing CVD morbidly and mortality [15]. Here, we found the 
number of hypertensive patients was reached to 13,036 (42.85\%) from 4,809 (15.81\%) and this raise has been observed in all age group, ranging from $22.2 \%$ in $20-29$ age group to $30.2 \%$ in $50-59$ years old subjects. A lower increased rate has been reported in other countries; $22.4 \%$ in China ( $>18$ years) [16], $13.7 \%$ in the USA (> 20 years) [17], and $6-11 \%$ in Sweden (25-74 years) [15], which indicates the number of people with SBP 130-140 or DBP 80-90 was higher in Iran and this country would receive greater benefit about reducing the burden of CVD in the future by implementation of ACC/AHA guideline. Moreover, a noticeable shift of HTN prevalence has been observed among male population. Although about equal number of male and female were diagnosed with HTN previously (17.79\% vs $14.70 \%$ ), a more significant increased rate has been found in the male sex after applying the 2017 guideline $(50.30 \%$ vs $38.70 \%$ ). This transformation is more correlated with the higher rate of CVD mortality among male population in Iran, which indicates the necessity of further interventional strategies in this gender.

According to the JNC8 guideline, we found $45.94 \%$ of the hypertensive subjects were aware of HTN, which is lower than the previous awareness estimates in Iran (59.2\%) [4]. After adopting the 2017 ACC/AHA, the awareness rate dropped to $22.77 \%$, which was more noticeable in the $40-49$ age group (21.65\%). Since $45.36 \%$ of this age group are now classified under the HTN category, a substantial call to action for public awareness is warranted. Moreover, the HTN treatment strategy has been revised in 2017 ACC/AHA and those with SBP 130-139 or DBP of 80-89 were also recommended to received antihypertension treatment if they are +65 years older, or the clinical CVD or 10-year atherosclerotic cardiovascular disease risk is $10 \%$ or greater [4]. Following this guideline, many high-risk adults might be protected against the development of HTN-related chronic diseases. However, the number of people who need anti-HTN treatment has been expected to increase from 8.1 million to 15.6 million in the USA and from 74.5 million to 129.8 million in China [6], which may impose a considerable impact on the health system in these countries. In this study, 4738 (15.53\%) of total sample were eligible to receive HTN therapy based on JNC8. After ACC/AHA, additional 1724 subjects with SBP 130-139 or DBP of 80-89 and history of CVD or DM become candidate for pharmacological interventions, which increase the treatment demand to $21.18 \%$. However, our study was limited to people aged 65 and younger; therefore, estimating the real impact of treatment in our population was not feasible. Another recent study conducted in Iran estimated that the number of adults met the criteria for treatment with antihypertensive medication increased from 13.4 million to 17.2 million [5]. Although the increased prevalence of HTN was more noticeable in Iran, the treatment burden in this country was lower compared to China \& the USA, which might be due to smaller population of $65+$ years old in Iran. Therefore, the impact of treatment costs on the health system under 2017 ACC/AHA guideline seems to be acceptable. However, the HTN control in Iran is relatively poor. Among those who were eligible for pharmacologic intervention, $19.40 \%$ take anti-HTN medication, which controlled the HTN in $60.72 \%$. Under ACC/AHA guideline, the blood pressure $<130 / 80 \mathrm{~mm} \mathrm{Hg}$ was considered as the target cut-off; therefore, the control rate was drop by $31.78 \%$ and reached to $28.94 \%$, which highlight a great need to expand the public health infrastructure for further managing the substantial increase in the public health burden of HTN.

Considering the 2017 ACC/AHA guideline, 6574 participants younger than 65 years old have been additionally diagnosed with HTN, who might benefit from earlier interventions. Since these group of 
people are not eligible for pharmacological interventions, modification of life style and nutrients diet is critical to reduce the risk of HTN-related disorders in the future. Although the salt intake was not an independent risk factor for HTN, the mean of sodium intake was estimated to be $8.74 \mathrm{gr} /$ day, which exceeds the current WHO recommendations ( $2 \mathrm{gr} /$ day(d) of Sodium, equivalent to $5 \mathrm{gr} / \mathrm{d}$ of salt) [18]. Based on 2017 ACC/AHA, we estimated about $79.38 \%$ of hypertensive people consume salt over the threshold, $75 \% .82$ need to lose extra weight, and $76.98 \%$ are required to do more physical activity.

We further evaluated the impact of 2017 ACC/AHA guideline on HTN-associated risk factors. All independent risk factors remained significant after implementation of the new guideline; however, the magnitude of the odds ratio reduced. Age was the strongest risk factor for HTN. Based on JNC8, the association between HTN and 60-65 aged group was 20.9-fold higher than HTN and 20-29 people; nevertheless, the association was reduced to 5.6-fold after adopting the updated version of the guideline. Similarity, we found a reduced rate of odds ratio in those with WHR $\geq 0.95$ and obese people, alcohol consumption and waterpipe smoking in manifesting the HTN in the future, which might influence the further awareness strategies in the populations about the effect of lifestyle risk factors on HTN development. Although this is a cross sectional-based study and a causal relationship cannot be inferred, it seems the association between HTN and history of DM, and CVD was reduced by $38 \%$, and $62 \%$, respectively after applying the of 2017 ACC/AHA guideline, which might indicate the benefit of 2017 guideline.

This is a cross-sectional based study; and a causal relationship cannot be inferred. Moreover, based on the HTN 2017 ACC/AHA those with SBP 130-139 or DBP of 80-89 were also recommended to received anti-hypertension treatment if they are +65 years older. However, our study was limited to people aged 65 and younger; and further study covering all range group is required to estimate the real impact of treatment after implementation of new guideline.

\section{Conclusion}

In the ACC/AHA guideline, a higher number of individuals with the pre-hypertension condition had been shifted into the hypertension category and the level of awareness, treatment, and control was dramatically fallen, which highlight a great need to expand the public health infrastructure for further managing the substantial increase in the public health burden of hypertension.

\section{Abbreviations}

\section{HTN}

Hypertension

CVD

cardiovascular diseases

JNC8

eighth report of the joint national committee 
SBP

systolic blood pressure

DBP

diastolic blood pressure

ACC

American College of Cardiology

AHA

American Heart Association

KCHS

Khuzestan Comprehensive Health Study

FBS

fasting plasma glucose levels

BMI

Body mass index

WHR

Waist-to-hip ratio

MCA

multiple correspondence analysis

MET

Metabolic equivalent of task

IPAQ

international physical activity questionnaire

HDL

high-density lipoproteins cholesterol

FFQ

food frequency questionnaire

SD

standard deviation

Declarations

Ethics approval and consent to participate

Not applicable

Consent for publication

Not applicable

Availability of data and materials

The datasets from the current study are included within the article. 


\section{Competing interests}

The authors declare that the research was conducted in the absence of any commercial or financial relationships that could be construed as a potential conflict of interest.

\section{Funding}

The project was approved by the National Institute for Medical Research Development, Iran

\section{Author contributions}

FS: concept, design, data analysis, interpretation, writing the article, critical reviews, final approval

BCh: data collection, critical reviews, final approval

ZM: data collection, critical reviews, final approval

SS: interpretation, critical reviews, final approval

SM: data analysis, critical reviews, final approval

ZR: data collection, critical reviews, final approval

LD: data collection, critical reviews, final approval

YP: data collection, critical reviews, final approval

FA: data collection, critical reviews, final approval

MN: data collection, critical reviews, final approval

SAM: data collection, critical reviews, final approval

AKSh: concept, design, interpretation, critical reviews, final approval

HP: concept, design, interpretation, critical reviews, final approval

\section{Acknowledgement}

Not applicable

\section{References}

1. Lawes CM, Vander Hoorn S, Rodgers A. Global burden of blood-pressure-related disease, 2001. Lancet. 2008;371:1513-8. doi:10.1016/s0140-6736(08)60655-8. 
2. Kearney PM, Whelton M, Reynolds K, Muntner P, Whelton PK, He J. Global burden of hypertension: analysis of worldwide data. Lancet. 2005;365:217-23. doi:10.1016/s0140-6736(05)17741-1.

3. Hernandez-Vila E. A review of the JNC 8 Blood Pressure Guideline. Tex Heart Inst J. 2015;42:226-8. doi:10.14503/thij-15-5067.

4. Carey RM, Whelton PK. Prevention. Detection, Evaluation, and Management of High Blood Pressure in Adults: Synopsis of the 2017 American College of Cardiology/American Heart Association Hypertension Guideline. Ann Intern Med. 2018;168:351-8. doi:10.7326/m17-3203.

5. Mahdavi M, Parsaeian M, Mohajer B, Modirian M, Ahmadi N, Yoosefi M, et al. Insight into blood pressure targets for universal coverage of hypertension services in Iran: the 2017 ACC/AHA versus JNC 8 hypertension guidelines. BMC Public Health. 2020;20:347. doi:10.1186/s12889-020-8450-1.

6. Khera R, Lu Y, Lu J, Saxena A, Nasir K, Jiang L, et al. Impact of 2017 ACC/AHA guidelines on prevalence of hypertension and eligibility for antihypertensive treatment in United States and China: nationally representative cross sectional study. BMJ. 2018;362:k2357. doi:10.1136/bmj.k2357.

7. Abariga SA, Khachan H, Al Kibria GM. Prevalence and Determinants of Hypertension in India Based on the 2017 ACC/AHA Guideline: Evidence from the India National Family Health Survey. Am J Hypertens. 2020;33:252-60. doi:10.1093/ajh/hpz181.

8. Kunes J, Zicha J. The interaction of genetic and environmental factors in the etiology of hypertension. Physiol Res. 2009;58(Suppl 2):33-41.

9. Lelong H, Blacher J, Baudry J, Adriouch S, Galan P, Fezeu L, et al. Individual and Combined Effects of Dietary Factors on Risk of Incident Hypertension: Prospective Analysis From the NutriNet-Santé Cohort. Hypertension. 2017;70:712-20. doi:10.1161/hypertensionaha.117.09622.

10. Saadat S, Yousefifard M, Asady H, Moghadas Jafari A, Fayaz M, Hosseini M. The Most Important Causes of Death in Iranian Population; a Retrospective Cohort Study. Emerg (Tehran). 2015;3:16-21.

11. Bundy JD, Li C, Stuchlik P, Bu X, Kelly TN, Mills KT, et al. Systolic Blood Pressure Reduction and Risk of Cardiovascular Disease and Mortality: A Systematic Review and Network Meta-analysis. JAMA Cardiol. 2017;2:775-81. doi:10.1001/jamacardio.2017.1421.

12. Shen $L, M a H, X i a n g ~ M X$, Wang JA. Meta-analysis of cohort studies of baseline prehypertension and risk of coronary heart disease. Am J Cardiol. 2013;112:266-71. doi:10.1016/j.amjcard.2013.03.023.

13. Huang $Y$, Wang S, Cai $X$, Mai W, Hu Y, Tang H, et al. Prehypertension and incidence of cardiovascular disease: a meta-analysis. BMC Med. 2013;11:177. doi:10.1186/1741-7015-11-177.

14. Brunström M, Carlberg B. Association of Blood Pressure Lowering With Mortality and Cardiovascular Disease Across Blood Pressure Levels: A Systematic Review and Meta-analysis. JAMA Intern Med. 2018;178:28-36. doi:10.1001/jamainternmed.2017.6015.

15. Brunström M, Carlberg B, Lindholm LH. Perspective From Sweden on the Global Impact of the 2017 American College of Cardiology/American Heart Association Hypertension Guidelines: A "Sprint" Beyond Evidence in the United States. Circulation. 2018;137:886-8. doi:10.1161/circulationaha.118.033632. 
16. Li D, Zeng X, Huang Y, Lei H, Li G, Zhang N, et al. Increased Risk of Hypertension in Young Adults in Southwest China: Impact of the 2017 ACC/AHA High Blood Pressure Guideline. Curr Hypertens Rep. 2019;21:21. doi:10.1007/s11906-019-0926-y.

17. Muntner P, Carey RM, Gidding S, Jones DW, Taler SJ, Wright JT Jr, et al. Potential US Population Impact of the 2017 ACC/AHA High Blood Pressure Guideline. Circulation. 2018;137:109-18. doi:10.1161/circulationaha.117.032582.

18. Härtl G. WHO issues new guidance on dietary salt and potassium. Cent Eur J Public Health. 2013;21:16.

\section{Figures}

a

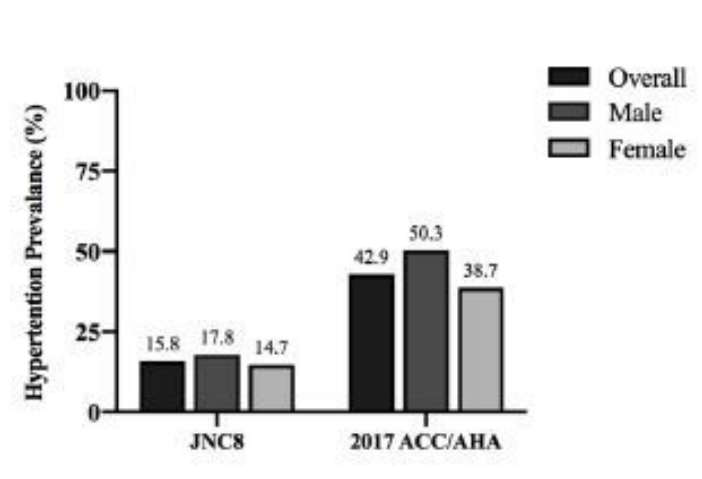

c b

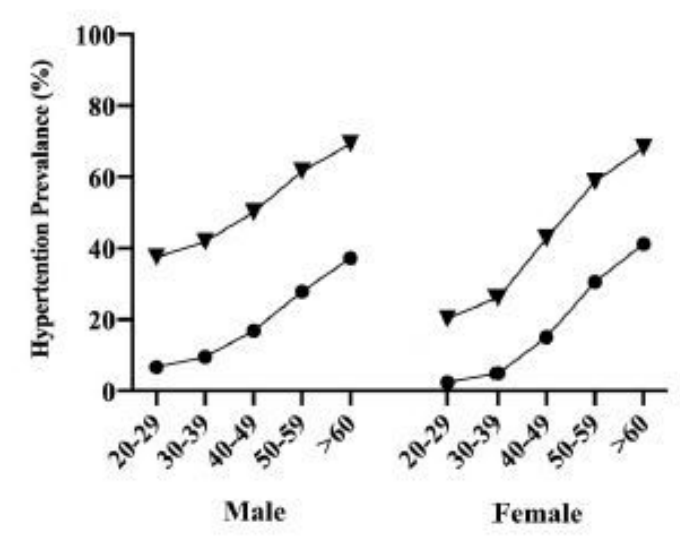

$\square$ JNC8
2017 ACCIAHA

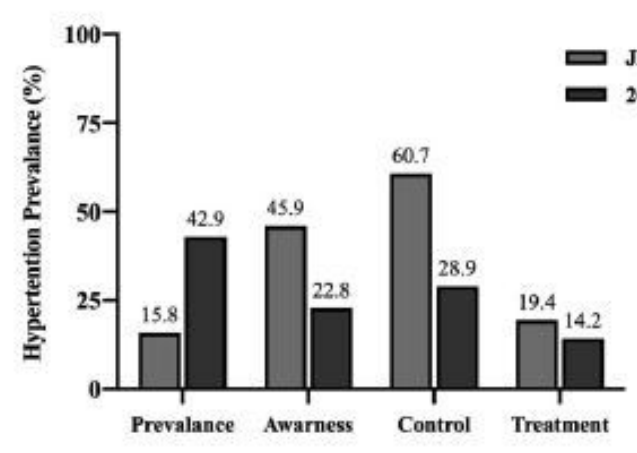

\section{Figure 1}

The impact of 2017ACC/AHA and JNC8 guidelines on the prevalence of hypertension in both sex (a), and different age groups (b), and awareness, control, and treatment of hypertension (c) in a population-based, cross-sectional study in Iran. 
Eligible for HTN treatment (Total: 4738)

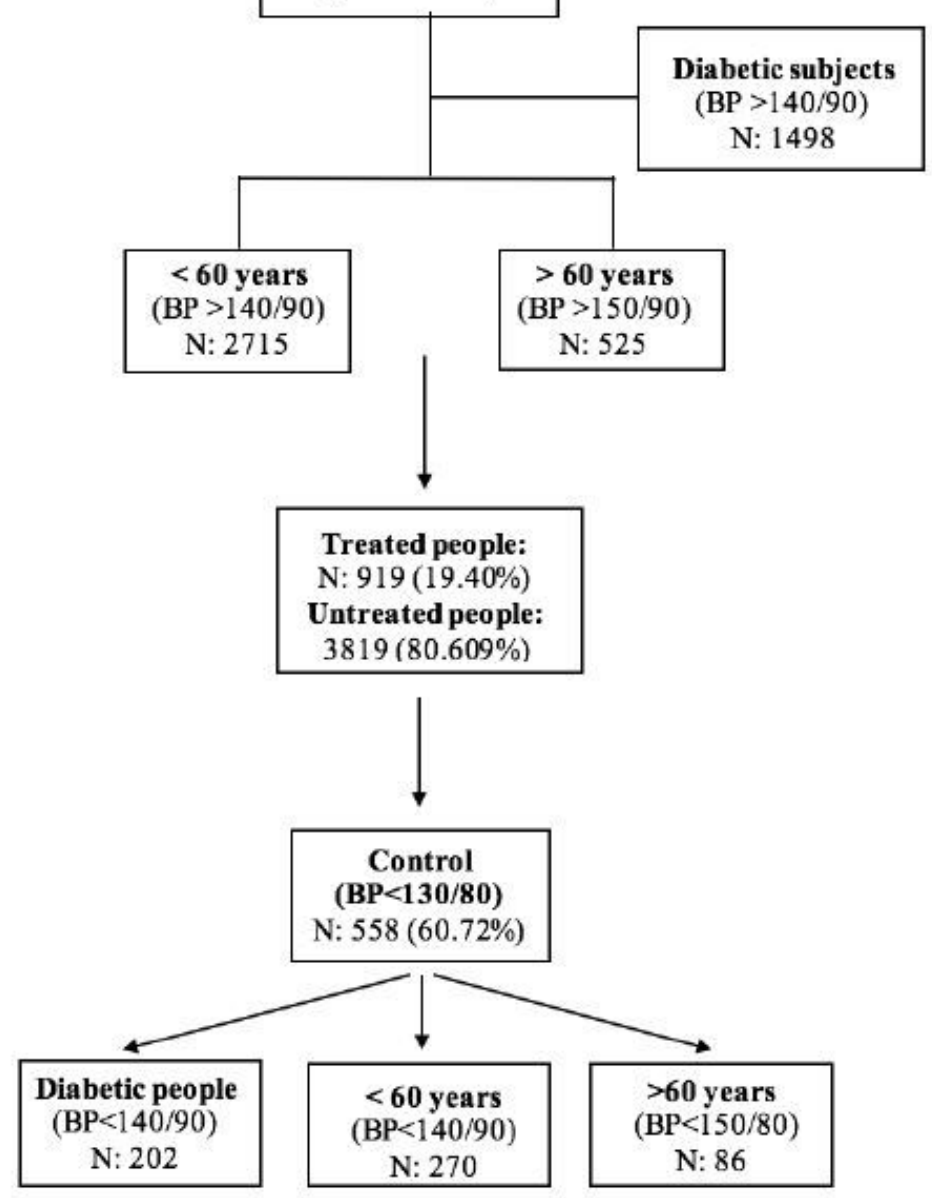

\section{ACC/AHA guideline}

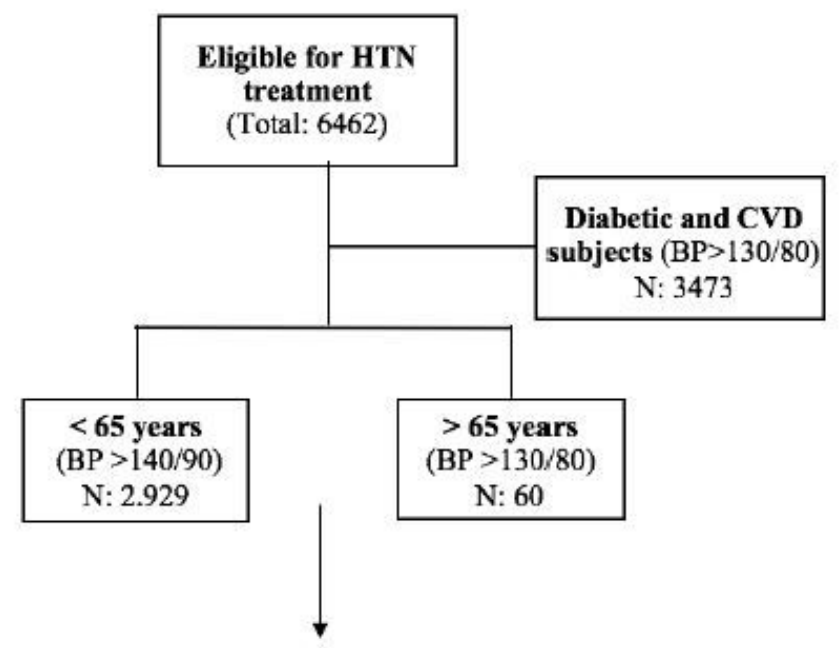

Treated people: N: 919 (14.22)

Untreated people: $5543(85.78)$

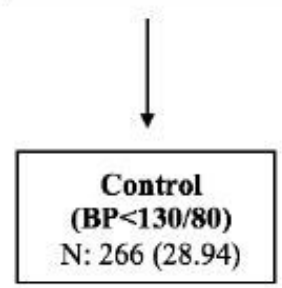

Figure 2

An estimation on the number of people who are eligible pharmacologic intervention, who were treated with antihypertensive agents and who had controlled hypertension based on JNC8 guideline and after implementation of 2017ACC/AHA guideline in a population-based, cross-sectional study in Iran. 